\title{
Park Plant Allocation Evaluation System Based on SBE
}

\author{
Lihan Wang ${ }^{\mathrm{a},{ }^{*}}$ \\ Academy of Fine Arts, China West Normal University, China \\ *,a52572523@qq.com
}

Keywords: park; plant landscape; configuration; SBE.

\begin{abstract}
For the systematic, comprehensive and theoretical analysis and evaluation of landscape design of garden plants, the SBE method is used to evaluate the plant in the landscape quality assessment, and the results of the evaluation in the different groups were obtained. The analysis of data shows that plant color richness, spatial level richness and plant species are the leading factors affecting the aesthetic quality of plant landscape. The paper tries to classify the collected samples in the process of evaluation, so as to get more accurate and reliable evaluation results. Based on the evaluation results, we analyzed the plant configuration landscape cases with high evaluation score, combined with the related theories of garden plants and art aesthetics, and made clear the local people's preferences for plant landscapes in urban parks, so as to provide scientific basis for plant landscape design in parks.
\end{abstract}

\section{Introduction}

The park green space is a city green place [1], which is open to the public, has professional planning and design and has certain activity facilities and garden layout, which provides residents with recreational activities and cultural sports activities. The quantity and quality of public green space in a city is an important symbol to measure the level of urban greening. The plant landscape space in the city can be divided into spot space, linear space and surface space [2]. As an important part of the green space system, the park plays an extremely important role in improving the ecological environment of the city and improving the people's quality of life. In the construction of parks, we often encounter how to build plant landscapes, plants and gardening elements, like landscape, roads and garden buildings.

Plant landscape is mainly the image expressed due to the natural vegetation, plant communities and plant individuals. It is transmitted to the cerebral cortex through people's sense and produces a real beauty feeling and imagination [3]. Plant landscapes also include artificial landscapes designed on plant materials. Plants are the main materials constitute landscape architecture and an indispensable part in landscape construction. Plant landscaping in modern urban parks is more concerned with the improvement of the current ecological environment and the function of service and use of the public. At the aesthetic level, other forms of art and related industries have a profound impact on modern plant landscaping [4]. It can be said that, under the influence of modern art, modern architecture and modern sculpture, the plant landscaping of urban parks allows different aesthetic orientations and design methods and has gradually developed into a diversified and open system. Therefore, the characteristics of plant landscaping in modern urban parks can be generally summarized as three points: advocate people-oriented idea, pay attention to ecology and sustainable development, and be deeply influenced by modern art. Under such a diversified development background, designers can draw on and refer to lessons from many new era's ideas and concepts, so as to produce colorful modern plant landscape [5]. 


\section{Evaluation Method of Plant Landscape Configuration}

\subsection{Evaluation Method}

Many studies show that people's aesthetic view is consistent with the common view, and under the guidance of the consistency, the average and common aesthetic view is regarded as an important measure standard of the quality of the scenery. People's judgment on scenery can be measured and evaluated through the scenery natural elements. It is widely recognized and accepted by people to use scene landscape to evaluate garden plant landscape and plant configuration [6]. There are a lot of scenic beauty measuring methods. This paper uses the SBE method in the psychological and physics method, and this method takes photo as the judgment and measurement media, regards the classified evaluation method as the basis, and makes the tested to score each landscape (often with pictures as a medium) in accordance with the given reference principle.

\subsection{Classification and Selection of Samples}

The basic research method is comparative law. By analyzing and comparing the differences and similarities of different research objects, we can see clearly the essential factors of things, or find the ways to solve practical problems from their gaps and differences. In this paper, combining the characteristics of plant landscape and the basic form of space, the samples for research and investigation, from the spatial perspective, are divided into the following types

Point independent plant landscaping: a point can set up many structural relations related to dots in space settings. Points can cause visual concentration, and all elements around it can appear the image and trend of point attracting. At the same time, the point has many expansionary features, which is manifested in the radiation of the surrounding things and space. Based on the characteristics of space, in the plant landscape, those are relatively independent and can be used as local spatial characteristics examples are classified as a class, namely point independent plant landscape [7].

Plant landscaping with linear sequence: the line is the movement trajectory of a point, and it is the superposition of points and points. The space defined by the line is a linear space, that is, a space with a wired nature. It has a visual sequence and continuity. In this study, the plant landscaping with obvious linear properties and more than 3 times the length was classified as a class [8]. Taking Yumen River Park in Taiyuan as the research object, based on the careful investigation of the greening plants in the park, and consulting the opinions of the insiders in detail, the main entrance area and quiet rest area of the park are selected as the main research objects.

\subsection{Photo Capture}

After investigating the plant landscape of Yumen River Park, photos of related plant landscapes were taken for testing and evaluation. According to the needs of research, plant landscape is divided into two types: spotted independent plant landscape and linear serial plant landscape. More than 50 photos of plant landscape were collected, and 28 color photographs were selected as media.

In shooting, we must keep consistency principle, try to make the pictures in the light intensity, photography angle and near future selection consistent, so as to increase the comparability between pictures and reduce man-made errors. At the same time, in order to reduce the number of errors caused by the sample, we try to choose the most representative picture as far as possible.

\subsection{Judge Choice}

The judges are divided into 3 groups, which consist of Table 1 as follows:

Table 1 . The composition of the judges

\begin{tabular}{|c|c|c|}
\hline Group name & Judge attribute & The number of people \\
\hline Gardening Specialty Group & Garden professional designer & 300 \\
\hline Ecology professional group & Staff with basic plant knowledge & 300 \\
\hline Public group & General public & 150 \\
\hline Total & & 750 \\
\hline
\end{tabular}

\subsection{Data Processing Method}

The questionnaire input data collected is input into office Excel software. The software function is used to solve SBE (Mathematical expectation) value and mutation coefficient (variance) for each landscape sample by judgment personnel in each group. In addition, the data curves are obtained by using the software function chart wizard. 


\section{Data Analysis}

\subsection{Evaluation and Analysis of Plant Landscape Samples}

The characteristics of plant configuration of the two types of landscape evaluation samples are show in Table 2 and Table 3 as follows:

Table 2. Configuration characteristics and SBE value of spot independent plant landscape

\begin{tabular}{|c|c|}
\hline Landscape & Plant configuration characteristics \\
\hline Landscape D1 & Landscape with central lawn to lilac, Hui and other enclosed \\
\hline Landscape D2 & Planting of spruce, Huang Yangqiu and forsythia Forsythia on the slope \\
\hline Landscape D3 & Pine, elm, and Picea meyeri stone combination landscape \\
\hline Landscape D4 & Plant landscape composed of red wood and Weigela combination \\
\hline Landscape D5 & Transparent space formed by lilac, Xunzi and willow \\
\hline Landscape D6 & Lilac scattered point planting \\
\hline Landscape D7 & Huang Cimei from the big lawn \\
\hline Landscape D8 & Potted plants, such as Rhododendron, placed in front of the entertainment equipment \\
\hline Landscape D9 & Prunustriloba composed of many trees under forest closed space \\
\hline Landscape D10 & Point space composed of lilac, wax, white enclosed building \\
\hline Landscape D11 & Huang Yangqiu and willow with pavilions \\
\hline Landscape D12 & Forest space composed of willow on the lawn scattered planting \\
\hline Landscape D13 & Forest space composed of elm trees scattered on the lawn \\
\hline Landscape D14 & Lilac, forsythia and flowering plum \\
\hline
\end{tabular}

Table 3. Configuration characteristics and its SBE value of linear sequence plant landscape

\begin{tabular}{|c|c|}
\hline Landscape & Plant configuration characteristics \\
\hline Landscape X1 & Space on the road formed by rose, lilac and so on \\
\hline Landscape X2 & Linear space formed by Jiaodong Euonymus scissor-shaped fence on the two sides of the road \\
\hline Landscape X3 & Tortuous path landscape formed by forsythia, Sabina and so on \\
\hline Landscape X4 & Scissor-shaped Jiaodong Euonymus, Ligustrum vicaryi and other park road landscape \\
\hline Landscape X5 & Landscape trees with obvious shade effect \\
\hline Landscape X6 & Landscape composed of lilacs planted in natural grassland with zigzag road \\
\hline Landscape X7 & Road endpoint landscape composed of Salix, Picea meyeri and Jiaodong Euonymus \\
\hline Landscape X8 & Roadside oil and pine forest landscape \\
\hline Landscape X9 & Road plant landscape composed of scissors-shaped ligustrum \\
\hline Landscape X10 & Road landscape formed by Jiaodong Euonymus and yellow BlackBerry \\
\hline Landscape X11 & Closed road space composed of lilac, Weigela, iris and gayfeather \\
\hline Landscape X12 & Plant landscape formed by a number of shears of Euonymus japonicus \\
\hline Landscape X13 & Road landscape composed of the lilacs plant \\
\hline Landscape X14 & Plant landscape composed of Populus \\
\hline
\end{tabular}

According to the results obtained from the questionnaire, the total SBE values evaluation diagrams of two types of landscape samples were drawn, such as Figure 1 and Figure 2.

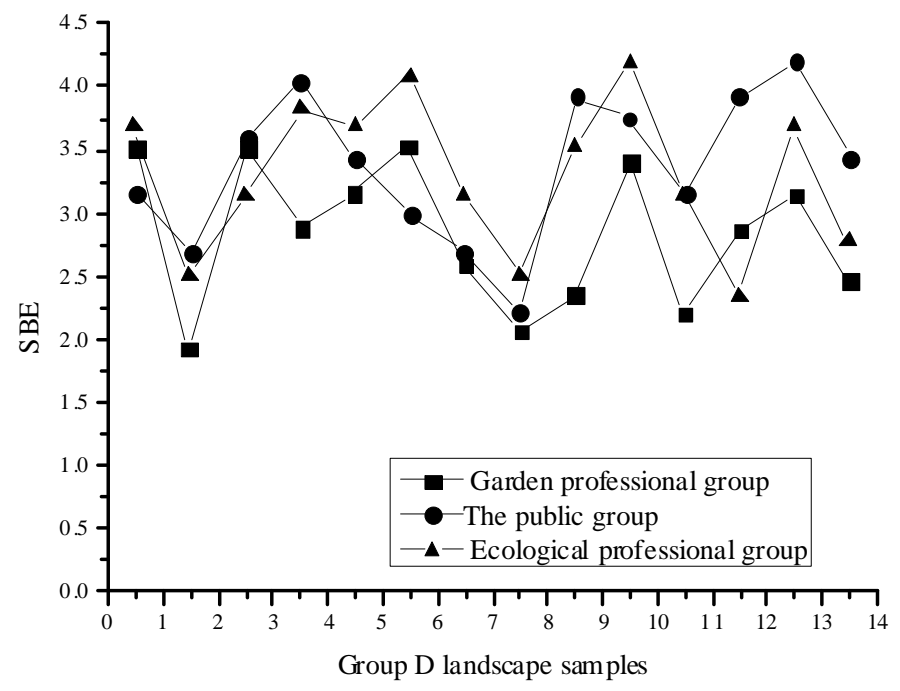

Figure 1. The data point line charts of point-like SBE freestanding plant landscape configuration 


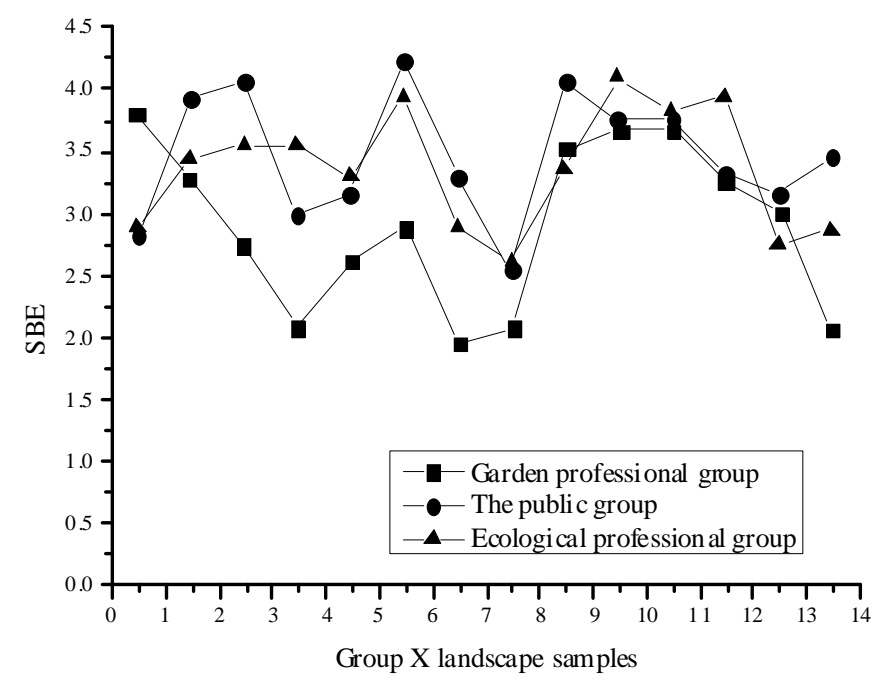

Figure 2. The data point line charts of SBE value of the landscape plant configuration of the linear sequence type

When each evaluation group used the SBE method to judge the two kinds of plant landscape, the SBE value of each evaluation sample was more consistent. From Figure 1 and Figure 2, we can see that the SBE value of the plant landscape samples is consistent with each other. The fluctuation of the data points of the three groups of judges is consistent, indicating that the SBE method can get stable and accurate evaluation results.

The SBE value given by evaluation group for point independent plant landscape in sample D1, D4, D6, D10, D13 and linear sequence plant landscape in sample X2, X9, X10, X11, X12 are high. From the above data, it can be seen that the common characteristics of these excellent landscape plants samples include: colorful plant species, plant landscape diversity and richness and so on. The beauty value of plant landscape is closely related to the variety of plant landscape, such as samples D1 and D10. The more the plant species is, the higher the score of plant landscape is. In addition, the level of plant planting is also directly related to the beauty degree of plant landscapes. That is, the higher the level of plant facade is, the higher the score of beautiful scenery is. For example, samples X2, X11 and sample X12 have more plant levels. Compared with the hard landscape such as plants and buildings, stone and other hard landscapes, the contrast of color and texture can also get better landscape effect, which is highly recognized by the judges.

For the plant landscape with lower SBE value given by judges, such as D2, D8 and D11 in point independent plant landscape and X7 and X8 in linear sequence plant landscape, through the analysis, we can get the following conclusions: these plant landscape gives people a messy feeling because of improper configuration structure or hierarchical confusion, color collocation also has the broken and not clear situation and so on. In addition, in this study, the biggest characteristic of plant landscape is the vitality. To create the ideal plant landscape, it not only needs the suitable tree, but also requires healthy plants. Only in this way can it give people a sense of beauty. For instance, the elm in samples D8 and X8 should be native species, which is suitable for the backbone of the foundation species. However, due to plant diseases and insect pests, excessive pruning and so on factors, it became the poor plant landscape.

\subsection{Evaluation and Analysis of the Evaluators}

When using SBE to evaluate, a notable factor is the judge. The judges' values, aesthetics, knowledge level and understanding degree of urban greening are different. Therefore, the judges, in the selection, should be as broad and representative as possible and coordinate with the evaluation methods so as to achieve the purpose of truly reflecting the beauty of the landscape. The mutation coefficient (C.V.) is used to describe the relative change and the change situation of the data around the mean value. We derive the mutation coefficient (namely variance) of the judges in the SBE method and get the same degree of convergence of the aesthetic orientation of the same group. 
The mutation coefficient of the results of two kinds of plant landscape evaluated by the SBE method was found in Table 4 and Table 5 by the garden professional group, the ecological professional group and the public group.

Table 4. Configuration characteristics and its SBE value of linear sequence plant landscape

\begin{tabular}{|c|c|c|c|}
\hline Plant landscape samples & Gardening professional group & Ecological professional group & Public group \\
\hline Landscape D1 & 1.3 & 2.5 & 1.5 \\
\hline Landscape D2 & 2.1 & 1.8 & 1.8 \\
\hline Landscape D3 & 1.3 & 2.9 & 1.5 \\
\hline Landscape D4 & 1.3 & 2.0 & 1.6 \\
\hline Landscape D5 & 1.3 & 0.9 & 2.6 \\
\hline Landscape D6 & 1.8 & 1.0 & 1.8 \\
\hline Landscape D7 & 2.2 & 1.8 & 1.8 \\
\hline Landscape D8 & 1.5 & 0.8 & 2.8 \\
\hline Landscape D9 & 2.0 & 1.8 & 1.0 \\
\hline Landscape D10 & 1.2 & 1.5 & 2.2 \\
\hline Landscape D11 & 2.0 & 1.3 & 2.1 \\
\hline Landscape D12 & 1.8 & 0.9 & 1.0 \\
\hline Landscape D13 & 1.8 & 2.5 & 0.9 \\
\hline Landscape D14 & 2.4 & 1.5 & \\
\hline
\end{tabular}

Table 5. Variation coefficient of the results of linear sequence plant landscape evaluation by SBE method among different groups

\begin{tabular}{|c|c|c|c|}
\hline Plant landscape samples & Gardening professional group & Ecological professional group & Public group \\
\hline Landscape X1 & 1.5 & 2.4 & 2.7 \\
\hline Landscape X2 & 2.1 & 1.2 & 1.6 \\
\hline Landscape X3 & 1.5 & 2.4 & 1.6 \\
\hline Landscape X4 & 1.5 & 1.3 & 3.1 \\
\hline Landscape X5 & 2.2 & 2.1 & 1.5 \\
\hline Landscape X6 & 2.4 & 1.5 & 2.2 \\
\hline Landscape X7 & 1.0 & 0.8 & 1.8 \\
\hline Landscape X8 & 1.5 & 2.2 & 1.9 \\
\hline Landscape X9 & 1.8 & 1.8 & 1.0 \\
\hline Landscape X10 & 1.4 & 1.5 & 1.6 \\
\hline Landscape X11 & 1.4 & 1.5 & 2.2 \\
\hline Landscape X12 & 2.1 & 1.5 & 3.0 \\
\hline Landscape X13 & 1.6 & 1.5 & 2.7 \\
\hline Landscape X14 & 1.5 & 1.3 & \\
\hline
\end{tabular}

According to Table 4 and Table 5, it can be seen that, when each group adopts SBE method to evaluate each landscape picture, its coefficient of variation is different, and the smaller its value is, the higher the group consistency is. It suggests that the aesthetic opinions of this group are basically in the same direction; otherwise, it is in the opposite direction.

\section{Conclusion}

From the coefficient of variation of each evaluation group, it can be seen that, the coefficient of variation of garden professional groups are small, which indicates that the knowledge of aesthetics for landscape is similar from those who have the same professional quality of judges from the angle of ecology and beauty. In the ecological professional group, with respect to the public group, has a good foundation for Botany. The evaluation results in this group, only the understanding variation coefficient for D3 group changes greatly, but aesthetic to other landscape still tends to be the same. For the data obtained from the public who have no basis for landscape and plant professional, we can see that coefficient of variation is great for the public group D8, X1, X12 and X13 landscape evaluation results. The reason maybe that, there is difference between the group for the plant landscape complex layer landscape richness, plant species richness and other knowledge, and the judges have some professional knowledge. As a result, when evaluating the configuration of plant landscape, as different coefficients of variation appear, the judges should try to refer to the evaluation results of smaller judges with certain professional knowledge. 


\section{References}

[1]. Moura F, Cambra P, Gonçalves A B. Measuring walkability for distinct pedestrian groups with a participatory assessment method: A case study in Lisbon [J]. Landscape \& Urban Planning, 2017, 157:282-296.

[2]. Zhou Q, Luo J. The Study on Evaluation Method of Urban Network Security in the Big Data Era [J]. Intelligent Automation \& Soft Computing, 2017:1-6.

[3]. Ding X, Chong X, Bao Z, et al. Fuzzy Comprehensive Assessment Method Based on the Entropy Weight Method and Its Application in the Water Environmental Safety Evaluation of the Heshangshan Drinking Water Source Area, Three Gorges Reservoir Area, China [J]. Water, 2017:329.

[4]. Feng C, Liu D, Chen Y, et al. Evaluation method of the conductive property of the electrode slurry in semi-solid lithium battery[J]. Gongneng Cailiao/journal of Functional Materials, 2017, 48(5):05011-05016.

[5]. Wang X G, Feng E S, Jiang C. A microplasticity evaluation method in very high cycle fatigue [J]. International Journal of Fatigue, 2017, 94:6-15.

[6]. Xia G, Luan T, Sun M. An Evaluation Method for Sortie Generation Capacity of Carrier Aircrafts with Principal Component Reduction and Catastrophe Progression Method [J]. Mathematical Problems in Engineering, 2017, (2017-5-28), 2017, 2017(4):1-10.

[7]. Pica A, Luberti G M, Vergari F, et al. Contribution for an Urban Geomorphoheritage Assessment Method: Proposal from Three Geomorphosites in Rome (Italy)[J]. Quaestiones Geographicae, 2017, 36(3).

[8]. Li J J, Wan C J, Niu J G, et al. Investigation on flexural toughness evaluation method of steel fiber reinforced lightweight aggregate concrete[J]. Construction \& Building Materials, 2017, 131:449-458. 